\title{
Associations among $\mathrm{FT}_{4}$ level, $\mathrm{FT}_{3} / \mathrm{FT}_{4}$ ratio, and non- alcoholic fatty liver disease in Chinese patients with hypopituitarism
}

\author{
Jinfeng Xiao ${ }^{1)}$ *, Chonggui Zhu ${ }^{1)}$, Xinxin Zhang ${ }^{1)}$, Longhao Sun²), Chang Gao ${ }^{1)}$, Xiaoyu Liang ${ }^{2)}$, \\ Qing $\mathrm{He}^{1)}$ and Ming Liu ${ }^{1)}$ \\ 1) Department of Endocrinology and Metabolism, Tianjin Medical University General Hospital, Tianjin, 300052, China \\ 2) Department of General surgery, Tianjin Medical University General Hospital, Tianjin, 300052, China
}

\begin{abstract}
Non-alcoholic fatty liver disease (NAFLD) is the most common hepatic metabolic disorder. Thyroid function is associated with NAFLD in different populations; however, little attention has been paid in patients with hypopituitarism. To analyze the association between thyroid function and NAFLD, we included 134 patients with hypopituitarism admitted to the Tianjin Medical University General Hospital between June 2013 and May 2019. Participants were divided into the NAFLD(-) and NAFLD $(+)$ groups based on abdominal ultrasonography findings. We evaluated 68 male and 66 female patients with hypopituitarism. The prevalence of NAFLD was $52.24 \%$. The NAFLD $(+)$ group had a significantly higher free triiodothyronine/free thyroxine $\left(\mathrm{FT}_{3} / \mathrm{FT}_{4}\right)$ ratio than the NAFLD $(-)$ group $(p=0.003)$. The NAFLD $(+)$ group showed significantly lower levels of $\mathrm{FT}_{4}$ and the growth hormone $(\mathrm{GH})$ than the NAFLD(-) group $(p=0.003$ and 0.016 , respectively). We observed an association of the $\mathrm{FT}_{4}$ level and $\mathrm{FT}_{3} / \mathrm{FT}_{4}$ ratio with NAFLD in the univariate model, which was nonsignificant after adjustment for metabolic parameters (BMI, HDL-C, triglycerides, serum uric acid, blood pressure, fasting glucose). To better understand the role of each metabolic parameters, we performed additional models for each of those predictors individually after adjustment for age and gender, the association between $\mathrm{FT}_{4}$ level and $\mathrm{FT}_{3} / \mathrm{FT}_{4}$ ratio lost significance after adjustment for HDL-C and TG, but not for other predictors. Our findings suggest that thyroid dysfunction may be crucially involved in NAFLD by regulating whole-body metabolism, especially lipid utilization. Therefore, sufficient thyroid hormone replacement therapy for patients with hypopituitarism is recommended from the early stage.
\end{abstract}

Key words: Hypopituitarism, Non-alcoholic fatty liver disease, Free thyroxine, Free triiodothyronine/free thyroxine ratio

\section{NON-ALCOHOLIC FATTY LIVER DISEASE}

(NAFLD) is the most common hepatic metabolic disorder. The increase in its prevalence is consistent with the increase in the worldwide prevalence of obesity and metabolic syndrome. The NAFLD prevalence in the general Asian population ranges from $15 \%$ to $40 \%$ [1]. NAFLD is a condition in which the fat deposited in the liver, due to causes other than excessive alcohol intake or other secondary causes, exceeds $5 \%$ of the liver's or hepatic weight [2]. NAFLD can progress to nonalcoholic steatohepatitis (NASH), with some cases progressing to liver cirrhosis and even hepatocellular carcinoma [3].

Submitted Aug. 26, 2021; Accepted Dec. 8, 2021 as EJ21-0536 Released online in J-STAGE as advance publication Jan. 14, 2022 Correspondence to: Qing He, MD, Department of Endocrinology and Metabolism, Tianjin Medical University General Hospital, No. 154 Anshan Road, Heping District, Tianjin, 300052, China. E-mail: heqing202108@163.com

*Jinfeng Xiao, Chonggui Zhu and Xinxin Zhang contributed equally to this work.
Thyroid hormones (THs) are involved in regulating body composition, lipid metabolism, and insulin resistance [4]. Hypothyroidism is associated with increased levels of low-density lipoprotein (LDL) cholesterol and triglycerides secondary to decreased activity of LDL receptors and clearance of triglyceride-rich lipoproteins $[5,6]$. Excessive triglyceride accumulation in the liver can lead to steatosis, which is a component of NAFLD or NASH [7]. Specifically, NAFLD is considered a hepatic characteristic of metabolic syndrome [8]. Therefore, it is speculated that THs may be involved in the pathogenesis of NAFLD.

Hypopituitarism is the deficiency of one or more hormones produced in the anterior pituitary gland, with the most common sequential pattern being the loss of growth hormone $(\mathrm{GH})$, follicle-stimulating hormone/luteinizing hormone, thyroid-stimulating hormone (TSH), and adrenocorticotropic hormone (ACTH), which may be accompanied by diabetes insipidus [9]. Patients with hypopituitarism, especially those with GH deficiency, 
have an increased prevalence of metabolic syndrome characterized by central obesity, dyslipidemia, and NAFLD, compared with that of the general population [10].

Hypopituitarism is one of the rare causes of NAFLD [11], which is often accompanied by secondary hypothyroidism due to insufficient TSH secretion. Previous studies have shown that THs may play a key role in the pathogenesis of NAFLD. However, the role of thyroid dysfunction in NAFLD progress in patients with hypopituitarism remains unclear. This study aimed to analyze the clinical characteristics and association of TH levels and the presence of NAFLD in Chinese patients with hypopituitarism.

\section{Materials and Methods}

\section{Participants and data collection}

We evaluated 178 patients with hypopituitarism who were admitted to the Department of Endocrinology and Metabolism of Tianjin Medical University General Hospital between June 2013 and May 2019. Hypopituitarism was diagnosed based on clinical manifestations, examination of pituitary-related hormones, and provocative tests. Diagnosis of hypopituitarism [12]: (1) Growth hormone deficiency (GHD) was diagnosed by a GH peak below $3 \mu \mathrm{g} / \mathrm{L}$ in the insulin tolerance test (ITT) in adults or below $5 \mu \mathrm{g} / \mathrm{L}$ in the ITT (or clonidine stimulation test in children); (2) Hypogonadotropic hypogonadism (HH) was defined by a reduction in the levels of total testosterone lower than the minimum limit of the reference values for the method used and for women was based on the presence of amenorrhea, reduced estradiol and inadequately normal or low FSH/LH concentrations; (3) Secondary hypothyroidism was diagnosed from serum free thyroxine $\left(\mathrm{FT}_{4}\right)$ level below the laboratory reference range in conjunction with a low, normal, or mildly elevated TSH; (4) Secondary adrenal insufficiency was considered in patients with serum cortisol levels lower than $5 \mu \mathrm{g} / \mathrm{dL}$ in an appropriate clinical context or cortisol peak lower than $18 \mu \mathrm{g} / \mathrm{dL}$ in the ITT; (5) Central diabetes insipidus was defined by the presence of high serum osmolarity ( $\left.>295 \mathrm{mOsm} / \mathrm{kgH}_{2} \mathrm{O}\right)$, while the corresponding urine osmolality should lower than $300 \mathrm{mOsm} /$ $\mathrm{kgH}_{2} \mathrm{O}$ in fluid deprivation tests. The exclusion criteria was as follows: (1) hypopituitarism secondary to functional pituitary tumors, including acromegaly, prolactinoma, TSH adenoma, and ACTH-dependent Cushing syndrome; (2) autoimmune diseases, including glucocorticoid-treated hypophysitis; (3) primary thyroid disease; (4) viral hepatitis and alcoholic fatty liver (weekly alcohol consumption $>21$ and 14 units for men and women, respectively); and (5) malignancy. Finally, 134 patients (66 females and 68 males; Fig. 1) were included in this study. There were no patients with liver cirrhosis. We retrospectively collected anthropometric indicators, laboratory tests, and abdominal ultrasonography findings. Based on the abdominal ultrasonography findings, the participants were divided into the NAFLD $(-)$ and NAFLD $(+)$ groups.

Data on patients' age, sex, race, smoking status, current alcohol consumption, cause and duration of hypopituitarism, laboratory findings, abdominal ultrasonography results, complications, comorbidies, and hormone replacement were collected from the standardized electronic inpatient record. Trained doctors measured height, weight, and blood pressure using a standard criterion. Body mass index (BMI) was calculated as weight (in kilograms) divided by height (in meters squared). The duration of hypopituitarism was calculated starting from the date of clinical diagnosis. The glucocorticoid replacement dose was expressed as the daily dose of hydrocortisone and the equivalent dose of $20 \mathrm{mg}$ hydrocortisone was $5 \mathrm{mg}$ prednisolone. The replacement dose of thyroid hormone was expressed as the daily dose of levothyroxine. Following an overnight fasting period of $\geq 10 \mathrm{~h}$, blood samples were obtained between 08:00 and 10:00 a.m. for biochemical analysis and examination of pituitary-related hormone levels and other parameters.

Further, based on the standardized criteria, NAFLD was diagnosed as hepatic steatosis according to the abdominal ultrasonography findings [13]. Abdominal ultrasonography, for assessment of hepatic steatosis, was performed for all participants by a trained operator who was blinded to the participants' clinical and laboratory characteristics, using a SIEMENS S3000 machine. NAFLD was diagnosed based on the presence of hepatic brightness, hepatorenal echo contrast, deep attenuation, and vascular blurring on ultrasonography [14].

\section{Statistical analysis}

For the descriptive analysis, categorical variables are expressed as the percentage and frequency. Numerical variables are expressed as the mean \pm standard deviation or medians and interquartile range, based on the sample distribution. Between group differences were evaluated using Student's $t$-tests and Mann-Whitney $U$ tests for non-categorical variables and the chi-square and Fischer's exact tests for categorical variables.

Multivariable logistic regression models were applied to assess the associations among metabolic parameters (including BMI, triglycerides, high-density lipoprotein cholesterol (HDL-C), and serum uric acid), GH levels, $\mathrm{FT}_{4}$ levels, $\mathrm{FT}_{3} / \mathrm{FT}_{4}$ ratio, and NAFLD presence. Subsequently, additional multivariable models adjusted as follows to estimate the effects of $\mathrm{FT}_{4}$ levels and the $\mathrm{FT}_{3} / \mathrm{FT}_{4}$ ratio on the odds of NAFLD presence were performed: 


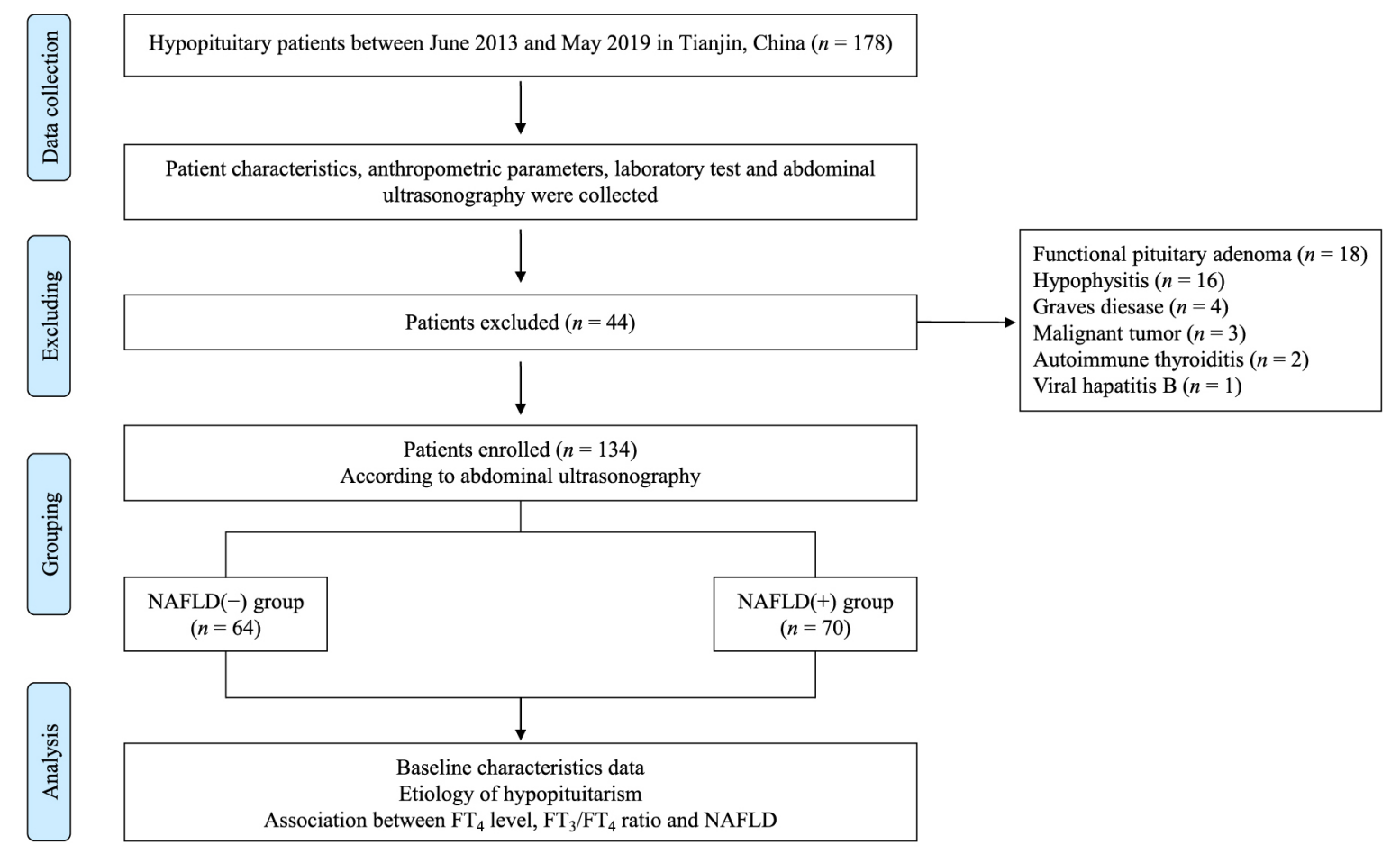

Fig. 1 Schematic overview of study data, calculations and analyses

$\mathrm{NAFLD}$, non-alcoholic fatty liver disease; $\mathrm{FT}_{3}$, free triiodothyronine; $\mathrm{FT}_{4}$, free thyroxine

Model 1: adjusted for age and sex

Model 2: adjusted for variables in model 1, duration, and smoking

Model 3: adjusted for variables in model 2 and daily dose of levothyroxine

Model 4: adjusted for variables in model 2 and metabolic parameters (BMI, HDL-C, triglycerides, serum uric acid, blood pressure, and fasting glucose)

To adjust for each metabolic parameter (BMI, HDL-C, triglycerides, serum uric acid, blood pressure, and fasting glucose), we used additional models for each of the aforementioned predictors after adjustment for age and sex.

Statistical analyses were performed using SPSS Statistics version 25.0 (IBM Corp., Armonk, New York). A $p$ value $<0.05$ was considered to indicate statistical significant.

\section{Study approval}

In this study, informed consent were not obtained because we used an electronic data set with consistent medical records from the Department of Endocrinology and Metabolism, which did not include personally identifiable information. The study protocol was approved by the Tianjin Medical University General Hospital Institutional Review Board with waiver of individual informed consent (approved number IRB2020YX-029-01).

\section{Results}

\section{Clinical characteristics of included participants}

Table 1 presents the baseline clinical characteristics of the participants according to the presence or absence of NAFLD. We performed a comparative analysis. There were $70(\mathrm{~F} / \mathrm{M}, 31 / 39)$ and $64(\mathrm{~F} / \mathrm{M}, 35 / 29)$ patients in the $\mathrm{NAFLD}(+)$ and $\mathrm{NAFLD}(-)$ groups, respectively. The overall prevalence of NAFLD was $52.24 \%$. The $\mathrm{NAFLD}(+)$ group had a significantly higher $\mathrm{FT}_{3} / \mathrm{FT}_{4}$ ratio than that of the NAFLD $(-)$ group $(0.34$ [0.27-0.42] vs. $0.28[0.22-0.36], p=0.003)$. Further, the NAFLD $(+)$ group had a significantly lower mean $\mathrm{FT}_{4}$ and $\mathrm{GH}$ levels than that of the NAFLD $(-)$ group $(9.42$ [7.27-12.70] vs. 12.34 [8.79-15.30], $p=0.003$ and 0.05 [0.05-0.11] vs. 0.07 [0.05-0.23], $p=0.016$; respectively). Additionally, the $\mathrm{NAFLD}(+)$ group had significantly higher BMI, alanine aminotransferase, aspartate aminotransferase, gamma-glutamyl transpeptidase, triglyceride (TG), uric acid (UA), daily dose of levothyroxine, and lower HDL$\mathrm{C}$ level than that of the NAFLD(-) group.

\section{Etiology of hypopituitarism and distribution of diagnosed hormone deficiency}

Table 2 presents the etiology and types of tumors in the included patients with hypopituitarism. There were between-group differences in the causes of hypopituitarism and tumor types ( $p=0.025$ and 0.034 , respectively). 
Table 1 Baseline characteristics of hypopituitary patients between group NAFLD $(+)$ and group NAFLD $(-)$

\begin{tabular}{|c|c|c|c|}
\hline Parameters & NAFLD $(-)$ & $\operatorname{NAFLD}(+)$ & $p$ value \\
\hline No. of participants & 64 & 70 & \\
\hline Male $n(\%)$ & $29(45.31)$ & $39(55.71)$ & 0.229 \\
\hline Age (yr) & $56.50(44.50-66.75)$ & $56.50(35.25-66.00)$ & 0.599 \\
\hline Duration (yr) & $3.00(0.23-10.00)$ & $2.00(0.75-7.50)$ & 0.926 \\
\hline BMI $\left(\mathrm{kg} / \mathrm{m}^{2}\right)$ & $23.34(20.46-25.58)$ & $26.90(25.01-29.09)$ & $0.000^{*}$ \\
\hline SBP (mmHg) & $120.00(110.00-140.00)$ & $120.00(110.00-135.50)$ & 0.756 \\
\hline $\mathrm{DBP}(\mathrm{mmHg})$ & $80.00(70.00-85.00)$ & $80.00(70.00-84.25)$ & 0.984 \\
\hline Smoking $n(\%)$ & $11(17.91)$ & $12(17.14)$ & 0.993 \\
\hline Daily dose of hydrocortisone & $20(10-40)$ & $20(10-30)$ & 0.166 \\
\hline Daily dose of levothyroxine & $25(0-50)$ & $25(12.50-62.50)$ & $0.035^{*}$ \\
\hline \multicolumn{4}{|l|}{ Routine blood tests } \\
\hline $\mathrm{WBC}\left(\times 10^{9} / \mathrm{L}\right)$ & $6.80 \pm 2.29$ & $7.33 \pm 2.24$ & 0.177 \\
\hline $\mathrm{RBC}\left(\times 10^{12} / \mathrm{L}\right)$ & $3.97 \pm 0.54$ & $4.12 \pm 0.41$ & 0.081 \\
\hline $\mathrm{Hb}(\mathrm{g} / \mathrm{L})$ & $121.03 \pm 14.71$ & $125.10 \pm 11.72$ & 0.080 \\
\hline $\operatorname{PLT}\left(\times 10^{9} / \mathrm{L}\right)$ & $247.49 \pm 69.74$ & $232.93 \pm 80.16$ & 0.269 \\
\hline FIB $(g / L)$ & $3.07 \pm 0.64$ & $2.86 \pm 0.78$ & 0.101 \\
\hline D-Dimer (ng/mL) & $482.50(285.00-812.00)$ & $394.50(257.00-585.25)$ & 0.123 \\
\hline \multicolumn{4}{|l|}{ Liver enzymes } \\
\hline $\operatorname{ALB}(g / L)$ & $42.00(38.00-45.00)$ & $42.00(40.00-45.50)$ & 0.495 \\
\hline $\operatorname{ALT}(\mathrm{U} / \mathrm{L})$ & $15.00(10.00-22.00)$ & $26.00(15.00-47.00)$ & $0.000^{*}$ \\
\hline $\operatorname{AST}(\mathrm{U} / \mathrm{L})$ & $18.00(15.00-25.00)$ & $23.00(17.00-33.00)$ & $0.004 *$ \\
\hline $\operatorname{ALP}(\mathrm{U} / \mathrm{L})$ & $66.00(52.00-86.50)$ & $63.00(51.00-90.50)$ & 0.837 \\
\hline GGT (U/L) & $16.00(12.50-23.00)$ & $30.00(22.00-63.00)$ & $0.000 *$ \\
\hline LDH (U/L) & $198.08 \pm 47.98$ & $216.52 \pm 55.86$ & $0.047^{*}$ \\
\hline TBIL $(\mu \mathrm{mol} / \mathrm{L})$ & $7.70(5.50-10.85)$ & $8.80(6.30-11.85)$ & 0.051 \\
\hline $\mathrm{DBIL}(\mu \mathrm{mol} / \mathrm{L})$ & $2.60(1.90-3.60)$ & $3.10(2.30-4.58)$ & $0.026^{*}$ \\
\hline \multicolumn{4}{|l|}{ Metabolic parameters } \\
\hline $\mathrm{TC}(\mathrm{mmol} / \mathrm{L})$ & $4.94(4.04-5.66)$ & $5.03(4.20-5.98)$ & 0.431 \\
\hline $\mathrm{TG}(\mathrm{mmol} / \mathrm{L})$ & $1.32(0.87-1.82)$ & $2.13(1.36-2.82)$ & $0.000^{*}$ \\
\hline LDL-C (mmol/L) & $2.93 \pm 1.09$ & $2.99 \pm 1.08$ & 0.752 \\
\hline HDL-C (mmol/L) & $1.165(0.87-1.43)$ & $0.97(0.79-1.16)$ & $0.002 *$ \\
\hline GLU (mmol/L) & $4.80(4.30-5.30)$ & $5.10(4.40-5.88)$ & 0.071 \\
\hline $\mathrm{UA}(\mu \mathrm{mol} / \mathrm{L})$ & $244.22 \pm 86.13$ & $316.06 \pm 109.52$ & $0.000^{*}$ \\
\hline Diabetes $n(\%)$ & $15(35.71)$ & 27 (64.29) & 0.059 \\
\hline Hypertension $n(\%)$ & $22(47.83)$ & $24(52.17)$ & 0.991 \\
\hline \multicolumn{4}{|l|}{ Pituitary related hormone } \\
\hline $\mathrm{TSH}(\mu \mathrm{IU} / \mathrm{mL})$ & $1.52(0.81-3.24)$ & $1.31(0.43-3.36)$ & 0.386 \\
\hline $\mathrm{FT}_{3}(\mathrm{pmol} / \mathrm{L})$ & $3.34 \pm 0.91$ & $3.25 \pm 0.70$ & 0.534 \\
\hline $\mathrm{FT}_{4}(\mathrm{pmol} / \mathrm{L})$ & $12.34(8.79-15.30)$ & $9.42(7.27-12.70)$ & $0.003 *$ \\
\hline $\mathrm{FT}_{3} / \mathrm{FT}_{4}$ & $0.28(0.22-0.36)$ & $0.34(0.27-0.42)$ & $0.003^{*}$ \\
\hline ACTH (pg/mL) & $17.60(10.62-28.30)$ & $15.80(8.90-26.23)$ & 0.509 \\
\hline Cor $(\mu \mathrm{g} / \mathrm{mL})$ & $10.72(4.43-21.75)$ & $6.78(2.88-16.33)$ & 0.076 \\
\hline $\mathrm{GH}(\mathrm{ng} / \mathrm{mL})$ & $0.07(0.05-0.23)$ & $0.05(0.05-0.11)$ & $0.016^{*}$ \\
\hline IGF-1 (ng/mL) & $45.70(26.80-120.00)$ & $64.90(30.60-114.00)$ & 0.492 \\
\hline
\end{tabular}

$* p<0.05$

Abbreviations: NAFLD, non-alcoholic fatty liver disease; BMI, body mass index; SBP, systolic blood pressure; DBP, diastolic blood pressure; WBC, white blood cells; RBC, red blood cells; Hb, hemoglobin; PLT, blood platelet; FIB, Fibrinogen; ALB, albumin; ALT, alanine aminotransferase; AST, aspartate aminotransferase; ALP, alkaline phosphatase; GGT, $\gamma$-glutamyltrans ferase; LDH, lacitc dehydrogenase; TBIL, total bilirubin; DBIL, direct bilirubin; TC, total cholesterol; TG, triglyceride; LDL-C, low density lipoprotein cholesterol; HDL-C, high density lipoprotein cholesterol; GLU, glucose; UA, uric acid; $\mathrm{TSH}$, thyroid stimulating hormone; $\mathrm{FT}_{3}$, free triiodothyronine; $\mathrm{FT}_{4}$, free thyroxine; ACTH, adrenocorticotropic hormone; Cor, cortisol; GH, growth hormone; IGF-1, insulin like growth factor 1 
Except for unclear causes, surgery was the most common cause of hypopituitarism. Based on the postoperative pathological results, non-functionging pituitary adenoma was the most common tumor type in the NAFLD $(+)$ group. Table 2 also shows the distribution of diagnosed hormone deficiency among the study participants. There was no significant between-group difference in the distribution of diagnosed hormone deficiency $(p=0.553)$. To confirm the physiological replacement of deficient hormones, following standard clinical practice, patients with hypopituitarism undergoing hormone replacement underwent regular clinical and biochemical tests. The prevalence of GHD was as high as $82.86 \%$ and $78.12 \%$ in $\mathrm{NAFLD}(+)$ and NAFLD $(-)$ groups, respectively. In addition, we found that the prevalence of adult-onset GHD was $61.43 \%$ and $56.25 \%$ in $\mathrm{NAFLD}(+)$ and $\operatorname{NAFLD}(-)$ groups, respectively while that of childhood-onset GHD was $21.43 \%$ and $21.88 \%$, respectively. There was no statistical difference between the two groups in the prevalence of adult-onset and childhood-onset GHD ( $p=$ 0.803). However, no patient in the study underwent $\mathrm{GH}$ replacement therapy.

\section{Risk factors for NAFLD in patients with hypopituitarism}

We performed logistic regression analysis to delineate the nature of the relationships among BMI, TG, HDL-C, $\mathrm{UA}, \mathrm{GH}, \mathrm{FT}_{4}, \mathrm{FT}_{3} / \mathrm{FT}_{4}$ ratio, and NAFLD as independent variables (Table 3 ). Univariate analysis revealed no association between GH levels and NAFLD. Compared with the NAFLD $(-)$ group, the NAFLD $(+)$ group had significantly lower $\mathrm{FT}_{4}$ levels and a higher $\mathrm{FT}_{3} / \mathrm{FT}_{4}$ ratio (Table 1). Univariate logistic analysis revealed a significant association of the $\mathrm{FT}_{4}$ level and $\mathrm{FT}_{3} / \mathrm{FT}_{4}$ ratio with NAFLD (Table 3), which remained significant after adjustment for age, sex, duration of hypopituitarism, smoking, and daily levothyroxine dose. However, the association was not significant after adjustment for metabolic parameters (Table 4).

Further analysis of each predictor revealed that the association between $\mathrm{FT}_{4}$ levels and $\mathrm{FT}_{3} / \mathrm{FT}_{4}$ ratio lost significance after adjustment for HDL-C and TG, but not other predictors (Table 5).

Table 2 Etiology of hypopituitarism, type of tumor and distribution of diagnosed hormone deficiency in patients with hypopituitarism $n(\%)$

\begin{tabular}{|c|c|c|c|}
\hline & $\operatorname{NAFLD}(-)(n=64)$ & $\operatorname{NAFLD}(+)(n=70)$ & $p$ value \\
\hline Etiology & & & $0.025^{*}$ \\
\hline Surgery & $18(28.12)$ & $32(45.71)$ & \\
\hline Radiotherapy & $2(3.12)$ & $6(8.57)$ & \\
\hline Surgery and radiotherapy & $3(4.69)$ & $5(7.14)$ & \\
\hline Vacuolar sella syndrome & $6(9.38)$ & $9(12.86)$ & \\
\hline Sheehan syndrome & $3(4.69)$ & $4(5.71)$ & \\
\hline Sellar lesions & $13(20.31)$ & $4(5.71)$ & \\
\hline Others & $19(29.69)$ & $10(14.29)$ & \\
\hline Type of tumor & & & $0.034^{*}$ \\
\hline Non-functioning pituitary adenoma & $22(34.38)$ & $25(35.71)$ & \\
\hline Craniopharyngioma & $5(7.81)$ & $14(20.00)$ & \\
\hline Intracranial germ cell tumor & $0(0.00)$ & $3(4.29)$ & \\
\hline Hypophyseal cyst & $5(7.81)$ & $1(1.43)$ & \\
\hline $\begin{array}{l}\text { Distribution of diagnosed hormone } \\
\text { deficiency }\end{array}$ & & & 0.553 \\
\hline Adrenal & $59(92.19)$ & $59(84.29)$ & \\
\hline Thyroid & $39(60.94)$ & $58(82.86)$ & \\
\hline Growth hormone & $50(78.12)$ & $58(82.86)$ & \\
\hline Gonadotrophines & $64(100)$ & $70(100)$ & \\
\hline Diabetes insipidus & $6(9.38)$ & $11(15.71)$ & \\
\hline
\end{tabular}




\section{Discussion}

NAFLD is considered the liver disease component of metabolic syndrome. Hypopituitarism, which induces a condition similar to metabolic syndrome, is associated with NAFLD. We found that the NAFLD prevalence in patients with hypopituitarism (52.24\%) was notably higher than that in the generation population $(25 \%$,

Table 3 Univariate logistic regression analysis of the association between NAFLD and related factors

\begin{tabular}{lccc}
\hline & OR & $95 \%$ CI & $p$ value \\
\hline BMI & 1.371 & $1.204-1.561$ & $0.000^{*}$ \\
TG & 1.798 & $1.230-2.627$ & $0.002 *$ \\
HDL-C & 0.206 & $0.068-0.618$ & $0.005^{*}$ \\
UA & 1.007 & $1.003-1.011$ & $0.000^{*}$ \\
$\mathrm{GH}$ & 0.673 & $0.372-1.219$ & 0.192 \\
$\mathrm{FT}_{4}$ & 0.862 & $0.784-0.947$ & $0.002 *$ \\
$\mathrm{FT}_{3} / \mathrm{FT}_{4}$ ratio & 1.048 & $1.014-1.083$ & $0.006^{*}$ \\
\hline
\end{tabular}

$\mathrm{FT}_{3} / \mathrm{FT}_{4}$ ratio $=\mathrm{FT}_{3} / \mathrm{FT}_{4} * 100$

$* p<0.05$

Abbreviations: NAFLD, non-alcoholic fatty liver disease; BMI, body mass index; TG, triglyceride; HDL-C, high density lipoprotein cholesterol; UA, uric acid; $\mathrm{GH}$, growth hormone; $\mathrm{FT}_{3}$, free triiodothyronine; $\mathrm{FT}_{4}$, free thyroxine reported in 2016) [15]; however, it was similar to that reported in Chinese adult patients with hypopituitarism (54\%) [16]. More attention should be paid to NAFLD caused by hypopituitarism since such cases can rapidly progress to cirrhosis even at a young age [17].

Our findings suggested that surgery, especially for nonfunctioning pituitary adenoma and craniopharyngioma, was the most common etiology of hypopituitarism with NAFLD. Surgical resection may damage the pituitary gland and hypothalamus. Since the hypothalamus is involved in regulating the appetite center and sympathetic nervous system, hypothalamic damage can also cause eating disorders and decreased sympathetic nerve activity, in addition to GH deficiency [18].

Disturbances in the endocrine hypothalamic-pituitary axis are associated with NAFLD, including GH deficiency, hypothyroidism, and hypogonadism [19]. The NAFLD prevalence in patients with hypopituitarismrelated GH deficiency is approximately $60 \%$. Additionally, GH supplementation therapy can reverse nonalcoholic steatohepatitis [20]. In this study, the prevalence of GHD was $82.86 \%$ in $\mathrm{NAFLD}(+)$ group. However, due to the difficulty in purchasing and high cost of growth hormone, most patients could not acquire it, and none of the patients with hypopituitarism, we included, received growth hormone therapy.

Table 4 Logistic regression for NAFLD outcome using $\mathrm{FT}_{4}$ level and $\mathrm{FT}_{3} / \mathrm{FT}_{4}$ ratio

\begin{tabular}{|c|c|c|c|c|c|c|c|c|}
\hline & \multicolumn{2}{|c|}{ Model 1} & \multicolumn{2}{|c|}{ Model 2} & \multicolumn{2}{|c|}{ Model 3} & \multicolumn{2}{|c|}{ Model 4} \\
\hline & OR & $p$ value & OR & $p$ value & OR & $p$ value & OR & $p$ value \\
\hline $\mathrm{FT}_{4}$ & 0.867 & $0.004 *$ & 0.859 & $0.003 *$ & 0.878 & $0.013^{*}$ & 0.881 & 0.074 \\
\hline $\mathrm{FT}_{3} / \mathrm{FT}_{4}$ ratio & 1.047 & $0.011 *$ & 1.057 & $0.005^{*}$ & 1.051 & $0.012^{*}$ & 1.016 & 0.543 \\
\hline
\end{tabular}

$\mathrm{FT}_{3} / \mathrm{FT}_{4}$ ratio $=\mathrm{FT}_{3} / \mathrm{FT}_{4} * 100$

Model 1: adjusted for age, gender

Model 2: adjusted for variables in model 1 and also adjusted for duration and smoking

Model 3: adjusted for variables in model 2 and also adjustesd for daily dose of levothyroxine

Model 4: adjusted for variables in model 2 and use of metabolic parameters (BMI, HDL-C, triglycerides, blood pressure, fasting glucose, uric acid)

$* p<0.05$

Abbreviations: NAFLD, non-alcoholic fatty liver disease; BMI, body mass index; HDL-C, high density lipoprotein cholesterol; $\mathrm{FT}_{3}$, free triiodothyronine; $\mathrm{FT}_{4}$, free thyroxine

Table 5 Logistic regression for NAFLD outcome with individual risk factors

\begin{tabular}{|c|c|c|c|c|c|c|c|c|c|c|c|c|}
\hline & \multicolumn{2}{|c|}{ BMI } & \multicolumn{2}{|c|}{ HDL-C } & \multicolumn{2}{|c|}{ TG } & \multicolumn{2}{|c|}{ SBP } & \multicolumn{2}{|c|}{ Glucose } & \multicolumn{2}{|c|}{ UA } \\
\hline & OR & $p$ value & OR & $p$ value & OR & $p$ value & OR & $p$ value & OR & $p$ value & OR & $p$ value \\
\hline $\mathrm{FT}_{4}$ & 0.852 & $0.004 *$ & 0.906 & 0.059 & 0.907 & 0.067 & 0.859 & $0.003 *$ & 0.854 & $0.003 *$ & 0.897 & $0.040^{*}$ \\
\hline $\mathrm{FT}_{3} / \mathrm{FT}_{4}$ & 1.048 & $0.025^{*}$ & 1.029 & 0.134 & 1.027 & 0.168 & 1.048 & $0.011^{*}$ & 1.045 & $0.021 *$ & 1.024 & 0.211 \\
\hline
\end{tabular}

$\mathrm{FT}_{3} / \mathrm{FT}_{4}$ ratio $=\mathrm{FT}_{3} / \mathrm{FT}_{4} * 100$

All data were adjusted for age and gender

$* p<0.05$

Abbreviations: NAFLD, non-alcoholic fatty liver disease; BMI, body mass index; HDL-C, high density lipoprotein cholesterol; TG, triglyceride; $\mathrm{SBP}$, systolic blood pressure; $\mathrm{UA}$, uric acid; $\mathrm{FT}_{3}$, free triiodothyronine; $\mathrm{FT}_{4}$, free thyroxine 
Kang SJ et al. enrolled 76 adolescents and young adult patients with childhood-onset hypopituitarism and 74 controls matched by age and BMI. Among patients with hypopituitarism, a lower insulin-like growth factor-1 standard deviation score (SDS) was associated with steatosis [21]. In our study, the NAFLD $(+)$ group showed significantly lower GH levels than the NAFLD $(-)$ group. However, logistic analysis revealed no significant association between GH levels and NAFLD. The reason for different results from previous studies may be due to the different ages of the included study population. In addition, Zhu $\mathrm{H}$ et al. established age- and sex-specific reference ranges for serum IGF-I on a large scale in a Chinese population. The $+2 \mathrm{SD},+\mathrm{SD}$, median, $-\mathrm{SD}$, and $-2 \mathrm{SD}$ of the serum IGF-I levels in healthy Chinese adults were obtained for both sexes [22]. Since the population in our study was patients with hypothyroidism, the IGF-1 level of the majority of patients were lower than -2 standard deviations, the exact IGF-1 SDS could not be used to analyze the GH deficiency.

Sex hormones are related to the presence and severity of NAFLD. Mueller NT et al. [23] found that sex hormones were associated with distinct histologic features of NAFLD in children and adolescents. The levels of testosterone in men and women are different, and the levels of $\mathrm{LH}, \mathrm{FSH}$, and progesterone in females are affected by the menstrual cycle and menopause. Since the levels of sex hormones could not be analyzed in this study, we could not clarify the associations between sex hormones and NAFLD. Therefore, we speculate that other pituitary-related hormones could be involved in the pathophysiology of NAFLD in patients with hypopituitarism.

It has been demonstrated that a relationship existed between NAFLD and thyroid dysfunction [24]. Silveira et al. observed thyroid dysfunction in approximately $25 \%$ of patients with NAFLD [25]. A matched casecontrol study reported a two-fold higher risk of hypothyroidism in patients with hepatic steatosis than that in controls [26]. Numerous studies have shown an inverse relationship of serum TH levels with NAFLD since patients with clinical and subclinical hypothyroidism have an increased risk of NAFLD and vice versa [27-29]. A study confirmed that independent of insulin resistance, the $\mathrm{FT}_{3} / \mathrm{FT}_{4}$ ratio was a predictor of NAFLD in both patients with euthyroidism and hypothyroidism [30]. Local conversion of $\mathrm{T}_{4}$ to the active form $\mathrm{T}_{3}$ by type 2 iodothyronine deiodinase $\left(\mathrm{DIO}_{2}\right)$ is a key mechanism of thyroid hormone regulation. The $\mathrm{FT}_{3} / \mathrm{FT}_{4}$ ratio was evaluated as an indirect index for increased conversion of $\mathrm{FT}_{4}$ to $\mathrm{FT}_{3}$, reflecting deiodinase activity [31]. A crosssectional study has indicated that $\mathrm{FT}_{3} / \mathrm{FT}_{4}$ ratio is strongly associated with insulin resistance in patients with euthyroidism and hypothyroidism [32]. An increased $\mathrm{FT}_{3} / \mathrm{FT}_{4}$ ratio was associated with metabolic syndrome and some of its components, including obesity, high TG, fasting blood glucose levels, and reduced HDL$\mathrm{C}$ levels in individuals with normal thyroid function [33, 34]. The increase in the $\mathrm{FT}_{3} / \mathrm{FT}_{4}$ ratio could be the consequence of adaptation to adverse metabolic conditions including dyslipidemia, insulin resistance, and metabolic syndrome, which enhance the activity of $\mathrm{DIO}_{2}$. Thus, a higher $\mathrm{FT}_{3} / \mathrm{FT}_{4}$ ratio possibly consequent to altered DIO expression, could to some extent reflect an effect of altered thyroid hormone status on NAFLD [31].

These findings suggest that thyroid dysfunction could promote NAFLD development. Moreover, most patients with hypopituitarism present with secondary hypothyroidism. In our study, TH replacement therapy was performed in $97(72.39 \%)$ patients. We found that the $\mathrm{NAFLD}(+)$ group had significantly lower $\mathrm{FT}_{4}$ levels and a higher $\mathrm{FT}_{3} / \mathrm{FT}_{4}$ ratio than the NAFLD(-) group. Logistic regression analysis revealed an association of the $\mathrm{FT}_{4}$ level and $\mathrm{FT}_{3} / \mathrm{FT}_{4}$ ratio with the presence of NAFLD in patients with hypopituitarism. Our findings are consistent with previous reports in other populations.

Although there are various hypotheses regarding the pathogenesis of NAFLD, the relationship among NAFLD, hypothyroidism, and metabolic syndrome remains unclear. The association between thyroid dysfunction and NAFLD could be mediated by metabolic parameters, including BMI, HDL-C levels, TG levels, UA levels, blood pressure, and fasting glucose, since there was no significant association after adjustment for those variables. Further analysis of each predictor revealed that the association between $\mathrm{FT}_{4}$ level and $\mathrm{FT}_{3} / \mathrm{FT}_{4}$ ratio lost significance after adjustment for HDL$\mathrm{C}$ and TG, but not other predictors. This could be attributed to THs being crucially involved in regulating systemic metabolism and liver lipid utilization [35]. Hypothyroidism can promote NAFLD development by causing hyperlipidemia. $\mathrm{TH}, \mathrm{T}_{4}$, and the more biologically active form $T_{3}$ stimulate fatty acid (FA) $\beta$-oxidation and oxidative phosphorylation in the liver [36]. In the liver, crosstalk between $\mathrm{TH}$ receptors and peroxisome proliferator-activated receptor alpha activates the expression of genes involved in FA $\beta$-oxidation [37-39]. Hepatic autophagy of fat droplets and their conversion to free FA in fused autolysosomes (lipophagy) could be essential for TH-mediated FA $\beta$-oxidation [40]. Briefly, since THs are involved in regulating hepatic lipid utilization, hypothyroidism-induced NAFLD could involve an intrahepatic mechanism. Specifically, the TH signal is impaired, which reduces hepatic FA utilization, with FAs being instead esterified and accumulated as TGs [37, 39]. A clinical study on Asian male patients with diabetes and hepatic steatosis found that low-dose levothyroxine 
supplementation for 4 months could significantly reduce hepatic fat content on magnetic resonance spectroscopy [41].

To the best of our knowledge, this is the first study to evaluate the relationship of the $\mathrm{FT}_{4}$ level and $\mathrm{FT}_{3} / \mathrm{FT}_{4}$ ratio with NAFLD in patients with hypopituitarism. However, this study had some limitations. First, since hypopituitarism is a rare endocrine disease, the sample size of the study was small. Thus, our findings cannot be generalized to other populations. Second, liver biopsy is considered appropriate for accurate diagnosis of NAFLD; however, NAFLD was diagnosed using abdominal ultrasonography for the study participants. The reported sensitivity and specificity of ultrasound are 53-100\% and 77-98\%, respectively [42]. Third, we did not measure insulin levels; therefore, we could not evaluate the association between insulin resistance and NAFLD. Fourth, we could not collect data on the thyroid function and abdominal ultrasonography findings of the patients before hormone replacement therapy because most patients were not initially diagnosed with hypopituitarism. To exclude the effect of thyroid hormone replacement on NAFLD, we adjusted for the daily dose of levothyroxine in Model 3. Finally, since this was a retrospective cross-sectional study, we could not determine causal relationships. Therefore, prospective, multicenter, cohort studies and animal experiments are warranted to elucidate the pathophysiology of NAFLD in patients with hypopituitarism.

In summary, we observed a high prevalence of NAFLD among patients with hypopituitarism. Therefore, NAFLD should be closely monitored in patients with hypopituitarism, especially those who underwent cranial surgeries. Additionally, thyroid dysfunction may be crucially involved in NAFLD by regulating whole-body metabolism, especially lipid utilization. Therefore, sufficient TH replacement therapy for patients with hypopituitarism is recommended from the early stage.

\section{Acknowledgements}

This study was supported by the Natural Science Foundation of China (81702410), Natural Science Foundation of Tianjin (17JCQNJC11100) and Natural Science Foundation of Tianjin Medical University (2016KYZQ02).

\section{Disclosure}

The authors declared no conflict of interest.

\section{References}

1. Younossi Z, Tacke F, Arrese M, Chander Sharma B, Mostafa I, et al. (2019) Global perspectives on nonalcoholic fatty liver disease and nonalcoholic steatohepatitis. Hepatology 69: 2672-2682.

2. Chalasani N, Younossi Z, Lavine JE, Charlton M, Cusi K, et al. (2018) The diagnosis and management of nonalcoholic fatty liver disease: practice guidance from the American Association for the Study of Liver Diseases. Hepatology 67: 328-357.

3. Younossi Z, Stepanova M, Ong JP, Jacobson IM, Bugianesi E, et al. (2019) Nonalcoholic steatohepatitis is the fastest growing cause of hepatocellular carcinoma in liver transplant candidates. Clin Gastroenterol Hepatol 17: 748-755.e3.

4. Chubb SA, Davis WA, Davis TM (2005) Interactions among thyroid function, insulin sensitivity, and serum lipid concentrations: the Fremantle diabetes study. J Clin Endocrinol Metab 90: 5317-5320.

5. Iglesias P, Díez JJ (2007) Influence of thyroid dysfunction on serum concentrations of adipocytokines. Cytokine 40: 61-70.

6. Shin DJ, Osborne TF (2003) Thyroid hormone regulation and cholesterol metabolism are connected through Sterol Regulatory Element-Binding Protein-2 (SREBP-2). J Biol Chem 278: 34114-34118.

7. Cotter TG, Rinella M (2020) Nonalcoholic fatty liver dis- ease 2020: the state of the disease. Gastroenterology 158: 1851-1864.

8. Yki-Järvinen H (2014) Non-alcoholic fatty liver disease as a cause and a consequence of metabolic syndrome. Lancet Diabetes Endocrinol 2: 901-910.

9. Miljić D, Popovic V (2018) Metabolic syndrome in hypopituitarism. Front Horm Res 49: 1-19.

10. Rufinatscha K, Ress C, Folie S, Haas S, Salzmann K, et al. (2018) Metabolic effects of reduced growth hormone action in fatty liver disease. Hepatol Int 12: 474-481.

11. Singeap AM, Stanciu C, Huiban L, Muzica CM, Cuciureanu T, et al. (2021) Association between nonalcoholic fatty liver disease and endocrinopathies: clinical implications. Can J Gastroenterol Hepatol 2021: 6678142.

12. Fleseriu M, Hashim IA, Karavitaki N, Melmed S, Murad MH, et al. (2016) Hormonal replacement in hypopituitarism in adults: an Endocrine Society clinical practice guideline. J Clin Endocrinol Metab 101: 3888-3921.

13. Bellentani $\mathrm{S}$, Saccoccio G, Masutti F, Crocè LS, Brandi G, et al. (2000) Prevalence of and risk factors for hepatic steatosis in Northern Italy. Ann Intern Med 132: 112-117.

14. Ozturk A, Grajo JR, Gee MS, Benjamin A, Zubajlo RE, et al. (2018) Quantitative hepatic fat quantification in non-alcoholic fatty liver disease using ultrasound-based techniques: a review of literature and their diagnostic 
performance. Ultrasound Med Biol 44: 2461-2475.

15. Lonardo A, Byrne CD, Caldwell SH, Cortez-Pinto H, Targher G (2016) Global epidemiology of nonalcoholic fatty liver disease: meta-analytic assessment of prevalence, incidence, and outcomes. Hepatology 64: 1388-1389.

16. Yuan XX, Zhu HJ, Pan H, Chen S, Liu ZY, et al. (2019) Clinical characteristics of non-alcoholic fatty liver disease in Chinese adult hypopituitary patients. World $J$ Gastroenterol 25: 1741-1752.

17. Yang Y, Qi ZR, Zhang TT, Kang YJ, Wang X (2018) Rapidly progressive non-alcoholic fatty liver disease due to hypopituitarism. Report of 5 cases. Neuro Endocrinol Lett 39: 99-104.

18. Kodama K, Ichihara A, Seki Y, Ikarashi Y, Sagawa T, et al. (2020) Characteristics of NAFLD based on hypopituitarism. Can J Gastroenterol Hepatol 2020: 8814435.

19. Eslam M, George J (2020) Genetic contributions to NAFLD: leveraging shared genetics to uncover systems biology. Nat Rev Gastroenterol Hepatol 17: 40-52.

20. Takahashi Y, Iida K, Takahashi K, Yoshioka S, Fukuoka $\mathrm{H}$, et al. (2007) Growth hormone reverses nonalcoholic steatohepatitis in a patient with adult growth hormone deficiency. Gastroenterology 132: 938-943.

21. Kang SJ, Kwon A, Jung MK, Chae HW, Kim S, et al. (2021) High prevalence of nonalcoholic fatty liver disease among adolescents and young adults with hypopituitarism due to growth hormone deficiency. Endocr Pract 27: 1149-1155.

22. Zhu H, Xu Y, Gong F, Shan G, Yang H, et al. (2017) Reference ranges for serum insulin-like growth factor I (IGFI) in healthy Chinese adults. PLoS One 12: e0185561.

23. Mueller NT, Liu T, Mitchel EB, Yates KP, Suzuki A, et al. (2020) Sex hormone relations to histologic severity of pediatric nonalcoholic fatty liver disease. J Clin Endocrinol Metab 105: 3496-3504.

24. Chung GE, Kim D, Kim W, Yim JY, Park MJ, et al. (2012) Non-alcoholic fatty liver disease across the spectrum of hypothyroidism. J Hepatol 57: 150-156.

25. Silveira MG, Mendes FD, Diehl NN, Enders FT, Lindor KD (2009) Thyroid dysfunction in primary biliary cirrhosis, primary sclerosing cholangitis and non-alcoholic fatty liver disease. Liver Int 29: 1094-1100.

26. Liangpunsakul S, Chalasani N (2003) Is hypothyroidism a risk factor for non-alcoholic steatohepatitis. J Clin Gastroenterol 37: 340-343.

27. Guo Z, Li M, Han B, Qi X (2018) Association of nonalcoholic fatty liver disease with thyroid function: a systematic review and meta-analysis. Dig Liver Dis 50: 1153-1162.

28. Ittermann T, Haring R, Wallaschofski H, Baumeister SE, Nauck M, et al. (2012) Inverse association between serum free thyroxine levels and hepatic steatosis: results from the Study of Health in Pomerania. Thyroid 22: 568-574.

29. Huang B, Yang S, Ye S (2020) Association between thyroid function and nonalcoholic fatty liver disease in euthyroid type 2 diabetes patients. J Diabetes Res 2020: 6538208 .
30. Gökmen FY, Ahbab S, Ataoğlu HE, Türker BÇ, Çetin F, et al. (2016) FT3/FT4 ratio predicts non-alcoholic fatty liver disease independent of metabolic parameters in patients with euthyroidism and hypothyroidism. Clinics (Sao Paulo) 71: 221-225.

31. van den Berg EH, van Tienhoven-Wind LJ, Amini M, Schreuder TC, Faber KN, et al. (2017) Higher free triiodothyronine is associated with non-alcoholic fatty liver disease in euthyroid subjects: the Lifelines Cohort Study. Metabolism 67: 62-71.

32. Štěpánek L, Horáková D, Štěpánek L, Janout V, Janoutová J, et al. (2021) Free triiodothyronine/free thyroxine (FT3/FT4) ratio is strongly associated with insulin resistance in euthyroid and hypothyroid adults: a crosssectional study. Endokrynol Pol 72: 8-13.

33. Xu R, Huang F, Zhang S, Lv Y, Liu Q (2019) Thyroid function, body mass index, and metabolic risk markers in euthyroid adults: a cohort study. BMC Endocr Disord 19: 58.

34. Urrunaga-Pastor D, Guarnizo-Poma M, Moncada-Mapelli E, Aguirre LG, Lazaro-Alcantara H, et al. (2018) High free triiodothyronine and free-triiodothyronine-to-freethyroxine ratio levels are associated with metabolic syndrome in a euthyroid population. Diabetes Metab Syndr 12: $155-161$.

35. Sinha RA, Singh BK, Yen PM (2018) Direct effects of thyroid hormones on hepatic lipid metabolism. Nat Rev Endocrinol 14: 259-269.

36. Sinha RA, Bruinstroop E, Singh BK, Yen PM (2019) Nonalcoholic fatty liver disease and hypercholesterolemia: roles of thyroid hormones, metabolites, and agonists. Thyroid 29: 1173-1191.

37. Mullur R, Liu YY, Brent GA (2014) Thyroid hormone regulation of metabolism. Physiol Rev 94: 355-382.

38. Jackson-Hayes L, Song S, Lavrentyev EN, Jansen MS, Hillgartner FB, et al. (2003) A thyroid hormone response unit formed between the promoter and first intron of the carnitine palmitoyltransferase-Ialpha gene mediates the liver-specific induction by thyroid hormone. $\mathrm{J}$ Biol Chem 278: 7964-7972.

39. Liu YY, Heymann RS, Moatamed F, Schultz JJ, Sobel D, et al. (2007) A mutant thyroid hormone receptor alpha antagonizes peroxisome proliferator-activated receptor alpha signaling in vivo and impairs fatty acid oxidation. Endocrinology 148: 1206-1217.

40. Sinha RA, You SH, Zhou J, Siddique MM, Bay BH, et al. (2012) Thyroid hormone stimulates hepatic lipid catabolism via activation of autophagy. J Clin Invest 122: 24282438 .

41. Bruinstroop E, Dalan R, Cao Y, Bee YM, Chandran K, et al. (2018) Low-dose levothyroxine reduces intrahepatic lipid content in patients with type 2 diabetes mellitus and NAFLD. J Clin Endocrinol Metab 103: 2698-2706.

42. Esterson YB, Grimaldi GM (2018) Radiologic imaging in nonalcoholic fatty liver disease and nonalcoholic steatohepatitis. Clin Liver Dis 22: 93-108. 